\title{
Interleukin-33 promotes obstructive renal injury via macrophages
}

\author{
YANLEI LI ${ }^{1,2}$, JING LIU ${ }^{3}$, TING YU ${ }^{2}$, BINGDI YAN ${ }^{3}$ and HONGJUN LI ${ }^{1}$ \\ ${ }^{1}$ Health Management Medical Center, China-Japan Union Hospital of Jilin University, Changchun, Jilin 130033; \\ Departments of ${ }^{2}$ Clinical Laboratory and ${ }^{3}$ Respiratory and Critical Care Medicine, \\ The Second Hospital of Jilin University, Changchun, Jilin 130041, P.R. China
}

Received October 13, 2018; Accepted April 12, 2019

DOI: $10.3892 / \mathrm{mmr} .2019 .10324$

\begin{abstract}
Chronic kidney disease is the outcome of most kidney diseases, and renal fibrosis is a pathological process involved in the progression of these disorders. The role of interleukin (IL)-33 was previously investigated in fibrotic disorders affecting various organs, including liver, lungs and heart; however, its role in renal fibrosis remains unclear. Previous studies have demonstrated that macrophages are involved in obstructive renal injury. In the present study, the roles of IL-33 and macrophages on renal fibrosis were investigated using a mouse model of unilateral ureteral obstruction (UUO). Compared with non-obstructed kidneys, the expression levels of IL-33 and its receptor, interleukin 1 receptor like 1, increased after UUO. Furthermore, the infiltration of macrophages and the degree of renal fibrosis increased after treatment with IL-33. Additionally, the expression level of arginase-1, a marker of M2 macrophages, increased in renal tissue. After depletion of macrophages, the administration of exogenous IL-33 was not sufficient to reverse the reduction in fibrosis caused by elimination of these cells. Collectively, the present results suggested that IL-33 promoted renal fibrosis in UUO-induced renal injury by regulating macrophage polarization.
\end{abstract}

\section{Introduction}

Renal fibrosis is a pathological injury process involved in the progression of all chronic kidney diseases (CKDs) to end-stage renal disease. Renal fibrosis and the development of sclerotic lesions result in nephritic failure and a progressive decline in renal function (1). The high incidence of CKD has become a medical and public health problem worldwide (2). Therefore,

Correspondence to: Professor Hongjun Li, Health Management Medical Center, China-Japan Union Hospital of Jilin University, 126 Xiantai Street, Changchun, Jilin 130033, P.R. China

E-mail: lihongjun1960@126.com

Key words: interleukin-33, macrophage, obstructive kidney disease, interleukin 1 receptor like 1 understanding the pathophysiology of CKD is crucial for the development of novel drugs and therapies aimed to limit CKD progression $(3,4)$. Unilateral ureteral obstruction (UUO) in animals has been used as an in vivo model to study the mechanisms of renal diseases (5). UOO can lead to acute changes in renal function, inducing chronic renal structural damage and promoting the rapid development of renal interstitial fibrosis (6). Compared with other models, UOO has the advantage of limiting the lesions to one kidney, avoiding systemic toxic effects (7).

Obstructive renal injury leads to the formation of tubular glomeruli, proximal tubular cell loss, immune cell infiltration, collecting duct remodeling and interstitial fibrosis $(8,9)$. Neutrophils and macrophages infiltrate the kidneys in the early phase of obstructive renal injury and maintain their innate immune functions. These cells are involved in kidney injury by synthesizing reactive oxygen species, pro-inflammatory mediators and proteases (10). In contrast with the neutrophils that are rapidly removed from the injury site, macrophages persist during the recovery phase, and were suggested to contribute to fibrosis $(11,12)$.

Following recruitment to the damaged kidney, macrophages can differentiate into two different subtypes depending on the microenvironment: Classically activated (M1) and alternatively activated (M2) macrophages (13). Pro-inflammatory M1 macrophages differentiation is mediated by exposure to interferon- $\gamma$ or lipopolysaccharide, whereas anti-inflammatory M2 macrophages are stimulated by T helper type 2 cytokines, such as interleukin (IL)-4 and IL-10 (14,15). M2 macrophages express arginase-1 (ARG1), whereas M1 macrophages express inducible nitric oxide synthase (16). A previous study demonstrated that IL-33, a member of the IL-1 family, cooperates with other cytokines in promoting the transition from M0 macrophages to M2 (17).

Transforming growth factor (TGF)- $\beta 1$ stimulates fibroblasts to synthesize stress fibers and undergo further differentiation to become myofibroblasts (18). IL-13 induces fibrosis by stimulating the synthesis and activation of TGF- $\beta 1$, or by directly activating the synthetic and proliferative properties of fibroblasts, epithelial cells and smooth muscle cells (19). Therefore, TGF- $\beta 1$ and IL-13 are essential for developing kidney fibrosis by stimulating the synthesis of extracellular matrix proteins, particularly collagen. In vitro studies found 
that IL-33 can promote IL-13-mediated macrophage polarization to M2 phenotype (20).

IL-33 is an immunomodulatory cytokine (21). IL-33 is expressed in various organs, and it was found to have the characteristics of an alarmin, being able to promote pro-inflammatory responses (20). The interleukin 1 receptor like 1 (IL1RL1) receptor is a member of the Toll/IL-1 receptor superfamily, and IL-33 is its agonist (22). A previous study demonstrated that upregulation of the IL-33/IL1RL1 signaling pathway in the obstructed kidney may promote tubular cell injury and interstitial fibrosis (23). Additionally, a previous study suggested that M2 macrophages, rather than M1 macrophages, are involved in renal fibrosis (24). Li et al reported that mature (m)IL-33, via IL1RL1, enhanced bleomycin-induced pulmonary fibrosis (25). However, the role of IL-33 in kidney disease remains unclear. Therefore, in the present study, the relationship between IL-33 and macrophages was investigated, and their roles in the development of renal fibrosis were examined using a mouse model of UUO.

\section{Materials and methods}

Animals. Specific-pathogen free C57BL/6 male mice $(\mathrm{n}=63$; age, 6-8 weeks; weight, 20-25 g) were purchased from the Institute of Laboratory Animal Sciences (Beijing, China). All procedures were approved by The Animal Experimental Ethics Committee of Jilin University. Animals were housed in SPF level barrier environment and kept at $22^{\circ} \mathrm{C}$ room temperature, $45 \pm 10 \%$ humidity, with a 12 -h light/dark cycle and free access to food and water.

Surgical procedure. A previous study demonstrated that isoflurane may affect the function of immune cells (26). By contrast, Zhang et al identified that the effect of isoflurane on immune function was limited compared with intraperitoneal injections of chloral hydrate and sodium pentobarbital (27). Since UUO and sham surgery are rapid procedures, the induction of anesthesia was performed using the recommended concentration of $4 \%$ isoflurane and continuous inhalation of $2 \%$ isoflurane was selected as maintain anesthesia after induction, as previously reported $(14,28)$. For surgical procedures, mice were placed on a temperature-controlled operating table. A small incision was performed on the left flank of the mouse, and the intestines were gently displaced from the abdomen and covered with sterile saline-soaked gauze. The left ureter was exposed and ligated with a 6-0 silk suture (Johnson and Johnson). The intestines were placed back in the abdomen, and the muscle, fascia and skin were sutured with sterile surgical 4-0 silk (Johnson and Johnson). Prophylactic povidone-iodine (10\%) was applied to the abdominal wound. For the sham surgery group, surgery was performed as aforementioned; however, the ureter was not ligated. Renal tissue and blood were harvested after 1, 3, 5, $7,9,11$ and 14 days ( $n=6$ in the day 7 and 14 groups; $n=3$ in the other groups).

mIL-33 treatment. Mice were treated with IL-33 to investigate its effect on UUO-induced fibrosis $(n=6)$. In the present study, $1 \mathrm{mg}$ recombinant murine mIL-33 (PeproTech, Inc.) was injected intraperitoneally on the same day of UOO surgery, and 1 and 2 days after UUO surgery. Mice treated with mIL-33 were sacrificed on day 7 .

Macrophage depletion. Macrophage depletion was performed to test whether macrophages were involved in IL-33-mediated pro-fibrotic effects $(n=6)$. Clodronate liposomes enter the macrophage membranes via endocytosis, and intracellular clodronate induces macrophage apoptosis (29). Kidney macrophage depletion was achieved by intraperitoneal injection of $200 \mu \mathrm{l}(\sim 1 \mathrm{mg})$ clodronate (ClodLip BV) or control liposomes on the same day of UUO surgery $(11,30)$, and 3 days after UUO surgery. Mice were sacrificed on day 7.

Cytokine measurements. Serum levels of IL-33 and additional inflammatory cytokines, including IL-13 and TGF- $\beta 1$, were determined using commercial ELISA kits (Cusabio Biotech Co., Ltd.; IL-33 cat. no. CSB-E14393; IL-13 cat. no. CSB-E04602m; and TGF- $\beta 1$ cat. no. CSB-E04726m) according to the manufacturer's protocol. The experiments were performed three times.

Reverse transcription-quantitative PCR (RT-qPCR). Total renal RNA was extracted from a quarter of each animal's kidney using the Eastep Super Total RNA extraction kit (Promega Corporation), according to the manufacturer's protocol. RNA concentration was determined spectrophotometrically at 260 and $280 \mathrm{~nm}$. A total of $2 \mu \mathrm{g}$ RNA was used to perform RT using the All-in-One First-Strand cDNA Synthesis kit (GeneCopoeia, Inc.): $37^{\circ} \mathrm{C} 60 \mathrm{~min}$ for reverse transcription reaction, $85^{\circ} \mathrm{C}$ for $5 \mathrm{~min}$ for termination of reaction. The FastStart Universal SYBR Green master mix (Roche Diagnostics) was used for qPCR, performed on a Light Cycler 480 (Roche Diagnostics). Thermal cycling conditions were $95^{\circ} \mathrm{C}$ for $10 \mathrm{~min}$ followed by 40 cycles of $95^{\circ} \mathrm{C}$ for $15 \mathrm{sec}, 60^{\circ} \mathrm{C}$ for $30 \mathrm{sec}$. Double distilled water was used as the negative control for the target and housekeeping gene $\beta$-actin used as an internal control. The sequences of the primers used were as follows (25): IL13 forward, 5'-GAATCCAGGGCTACA CAGAAC-3' and reverse, 5'-AACATCACACAAGACCAGA CTC-3'; IL33 forward, 5'-ACTATGAGTCTCCCTGTCCTG-3' and reverse, 5'-ACGTCACCCCTTTGA AGC-3'; IL1RL1 forward, 5'-TCTGTGGAGTACTTTGTTCACC-3' and reverse, 5'-TCTGCTATTCTGGATACTGCTTTC-3'; TGF- $\beta 1$ forward, 5'-CCATGAGGAGCAGGAAGG-3' and reverse, 5'-ACAGCA AAGATAACAAACTCCAC-3'; collagen 3 forward, 5'-TCT CTAGACTCATAGGACTGACC-3' and reverse, 5'-TTCTTC TCACCCTTCTTCATCC-3'; ARG1 forward, 5'-AGTGTT GATGTCAGTGTGAGC-3' and reverse, 5'-GAATGGAAG AGTCAGTGTGGT-3', as previously reported.

Renal histology and immunohistochemical staining. The kidneys were dissected and cut into two parts. Each kidney was cut lengthwise and half of each kidney $(n=6)$ was fixed using $10 \%$ formalin for at least $24 \mathrm{~h}$ (room temperature; $20-24^{\circ} \mathrm{C}$ ). After fixation, the tissue was dehydrated with gradient ethanol, transparent with n-butanol and embedded with paraffin, and $5-\mu \mathrm{m}$ sections. Following xylene dewaxing and rehydration in descending ethanol series, the sections were stained with hematoxylin and eosin, and Masson's trichrome staining solution, to assess fibrosis. For 
immunohistochemistry, Xylene dewaxing and transparent; rehydration in descending ethanol series, then an additional antigen retrieval step was performed by heating samples in an EDTA-based buffer ( $\mathrm{pH} \mathrm{9.0)} \mathrm{at} 95-100^{\circ} \mathrm{C}$ for $15 \mathrm{~min}$. blocked with 5\% BSA (AR0004, Wuhan Boster Biological Technology, Ltd.) for $30 \mathrm{~min}$ at $37^{\circ} \mathrm{C}$. The primary antibodies used were anti-IL-33 (1:200; cat. no. AF3626; R\&D Systems, Inc.) and anti-adhesion $G$ protein-coupled receptor E1 (ADGRE1; 1:200; cat. no. MAB5580; R\&D Systems, Inc.). Samples were incubated with primary antibody for $10 \mathrm{~h}$ at $4^{\circ} \mathrm{C}$. The secondary antibodies used were Horseradish Peroxidase-conjugated Affinipure Rabbit Anti-Goat IgG (1:200; cat. no. SA00001-4; ProteinTech Group, Inc.) and Horseradish Peroxidase-conjugated Affinipure Goat Anti-Rat IgG (1:200; cat. no. SA00001-15; ProteinTech Group, Inc.). The secondary antibodies incubation was $37^{\circ} \mathrm{C}$ for $60 \mathrm{~min}$. Images were obtained using an Olympus BX51 microscope and DP2-BSW imaging system (Olympus Corporation). Masson's trichrome stained samples were analyzed using ImageJ software (National Institutes of Health, version 1.4.3.) and regions stained in blue were quantified. In total, 5 randomly selected fields of view were analyzed for each sample. IL-33-positive cells and macrophages (ADGRE1-positive cells) were counted in each microscopic field, and the mean number of positive cells per high-power field (magnification, x400) was calculated.

Western blot assay. Kidney tissue proteins were extracted using RIPA assay lysis buffer (cat.no.DE101; Beijing Transgen Biotech Co., Ltd.), and protein concentrations were determined using a bicinchoninic protein assay kit (cat. no. E162-01; Gene-star Biosolutions Co., Ltd.). Protein separation was performed by $10 \%$ SDS-PAGE. The separated proteins were transferred to a PVDF membrane (EMD Millipore) which was incubated with $5 \%$ non-fat milk in Tris buffer for $1 \mathrm{~h}$ at room temperature to block non-specific binding. The membranes were then incubated overnight at $4^{\circ} \mathrm{C}$ with a primary antibody against ARG1 (1:1,000; cat. no. 93668; Cell Signaling Technology, MA). The PVDF membrane was washed with TBST (\% 0.05 Tween20; cat. no. E175-01; Gene-star Co., Ltd.) three times, followed by incubation with a secondary horseradish peroxidase-labeled anti-rabbit immunoglobulin G (1:2,000; cat. no. SA00001-2; ProteinTech Group, Inc.) for $1 \mathrm{~h}$ at room temperature. Then the PVDF membrane was washed with TBST three times, 10 min each time. $\beta$-Tubulin (cat. no. HC101; Beijing Transgen Biotech Co., Ltd.) was used as a protein loading control, the membranes were incubated with primary antibody $\beta$-Tubulin overnight at $4^{\circ} \mathrm{C}$ and then processed for detection by ECL (cat. no. abs920; Absin Bioscience, Inc.), using an integrated chemiluminescence imaging system (ChemiScope 6000; Clinx Science Instruments Co., Ltd.) and ChemiCapturePAD V4.0.12.812 (Clinx Science Instruments Co., Ltd.) for detection and imaging.

Statistical analyses. All data are presented as the mean \pm SD from three independent experiments $(n=6$ in each experiment, except for the UUO and sham groups at days 1, 3, 5,9 and 11, where $n=3)$. Student's t-test was used to compare two groups. One-way ANOVA followed by Student-Newman-Keuls post hoc test was used to compare multiple groups. $\mathrm{P}<0.05$ was considered to indicate a statistically significant difference. Analyses were performed using GraphPad Prism software (version 5.0; GraphPad Software, Inc.).

\section{Results}

Protein expression levels of IL-33 and ILIRLI are increased in a mouse model of UUO. Hematoxylin and eosin, and Masson's trichrome staining showed increased inflammatory cell infiltration and collagen deposition in the kidney after UUO induction (Fig. 1A, B and D). The present data suggested that UUO mice exhibited renal inflammation and interstitial fibrosis.

Immunohistochemical analysis of renal tissue sections from sham and UUO mice suggested that renal tubular epithelial cells expressed high levels of IL-33 (Fig. 1C and E). Moreover, increased expression levels of IL-33 were detected in the UUO group compared with the sham surgery group. The mRNA expression level of collagen 3 was also assessed. The expression level of collagen 3 increased in UUO mice compared with the sham group (Fig. 1F). The serum level of IL-33 increased significantly in the UUO and sham operation groups 1 day after surgery (Fig. 1G). This effect may be due to the stimulation induced by the surgical trauma, which promoted the infiltration of local inflammatory cells and consequent secretion of IL-33. On the following days, the serum levels of IL-33 in the sham group were similar to day 0 , whereas the serum levels of IL-33 in the UUO group were significantly increased compared with the sham group. In addition, the expression levels of IL-33 and IL1RL1 in renal tissue were increased compared with the sham group at days 3, 5, 7, 9, 11 and 14 after surgery (Fig. $1 \mathrm{H}$ and I).

Recombinant mIL-33 exacerbates UUO-induced renal fibrosis and macrophage infiltration in mice kidneys. Mice were injected intraperitoneally with mIL-33 on the same day of UUO surgery. Sham-operated and UUO mice were used as control groups. Kidney tissues were harvested on day 7 . Exogenous mIL-33 significantly increased UUO-induced renal inflammation (Fig. 2A) and collagen deposition (Fig. 2B and D) compared with the sham surgery group. Treatment with IL-33 significantly increased the expression level of collagen compared with the UOO group ( $\mathrm{P}<0.05$; Fig. $2 \mathrm{~F})$.

IL-33 was previously identified to influence the phenotype and function of macrophages (31). In addition, ADGRE1 is a well-known macrophage marker (5). In the present study, to investigate the relationship between macrophages and IL-33 in obstructive renal injury, immunostaining of ADGRE1 was performed on renal tissue. The infiltration of ADGRE1 positive macrophages increased in UUO mice treated with recombinant $\mathrm{mIL}-33$ compared with the sham and UUO groups (Fig. 2C and E). The number of macrophages was higher in the renal tubule area compared with other regions (Fig. 2C).

UUO induces IL-33 production, which promotes renal fibrosis through macrophages, increasing the secretion of IL-13 and $T G F-\beta 1$. A previous study identified that IL-13 and TGF- $\beta 1$ are key cytokines required for the development of fibrosis (19). As mentioned above, IL-33 exacerbated UUO-induced renal fibrosis and the expression levels of these cytokines. However, macrophage depletion (Fig. 3A and D) decreased renal 

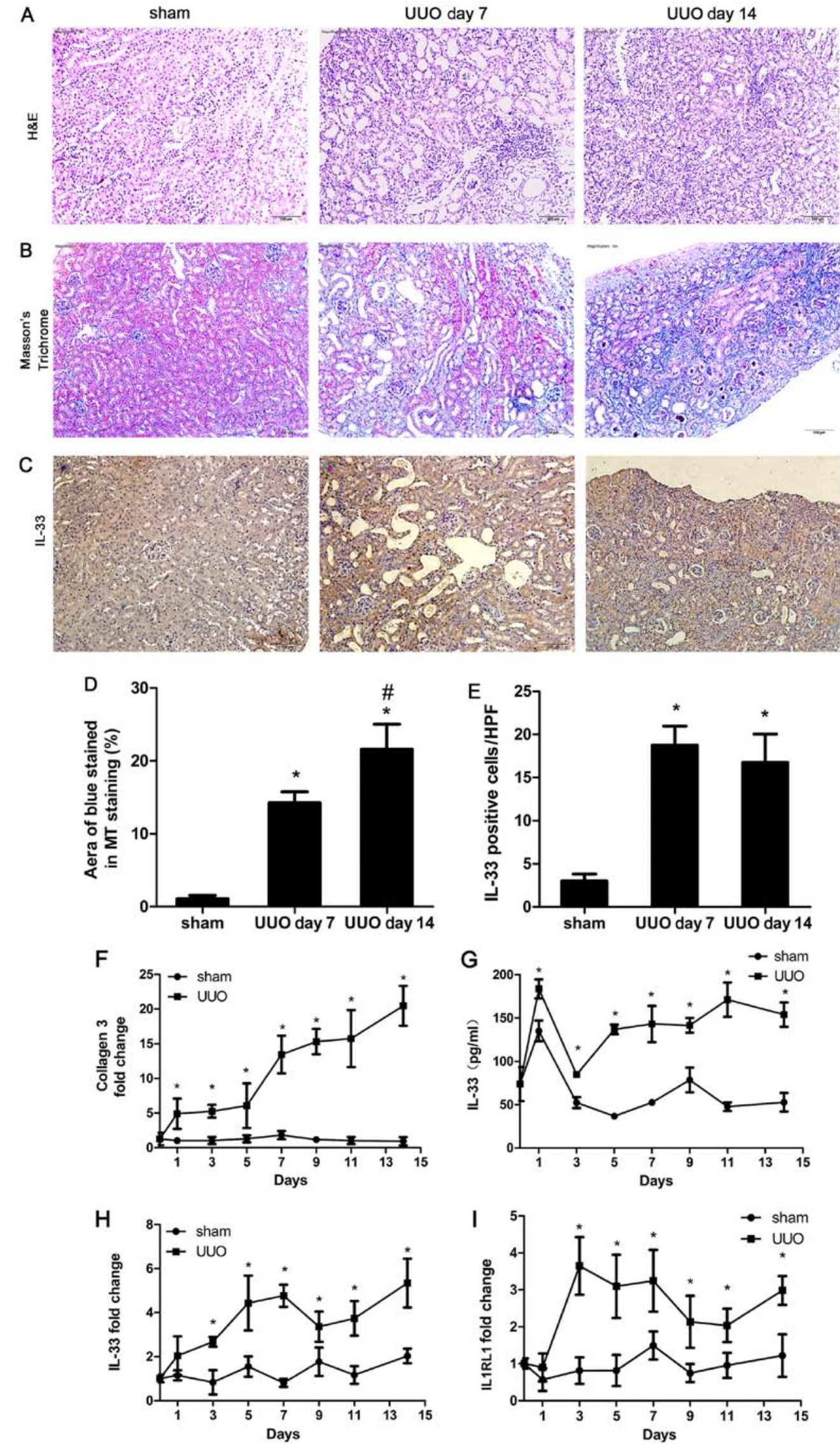

Figure 1. Protein expression levels of IL-33 and IL1RL1 in sham operated and UUO model mice. (A) H\&E staining showing renal inflammatory cell infiltration in UUO and sham operated mice. (B) Collagen stained by MT solution in UUO and sham operated mice. Fibrotic tissue is stained in blue. (C) Immunohistochemical staining of IL-33. (D) Quantification of MT staining results. (E) Quantification of IL-33-positive cells. (F) Collagen 3 relative mRNA expression levels. (G) Changes in IL-33 serum levels. (H) IL-33 relative mRNA expression levels. (I) IL1RL1 relative mRNA expression levels. Magnification, $x 200$. Data are expressed as the mean \pm SD from three independent experiments. ${ }^{*} \mathrm{P}<0.05$ vs. sham; ${ }^{\#} \mathrm{P}<0.05$ vs. UUO at day 7. IL, interleukin; UUO, unilateral ureteral obstruction; IL1RL1, interleukin 1 receptor like 1; MT, Masson's trichrome; HPF, high-power field; H\&E, hematoxylin and eosin. 
A
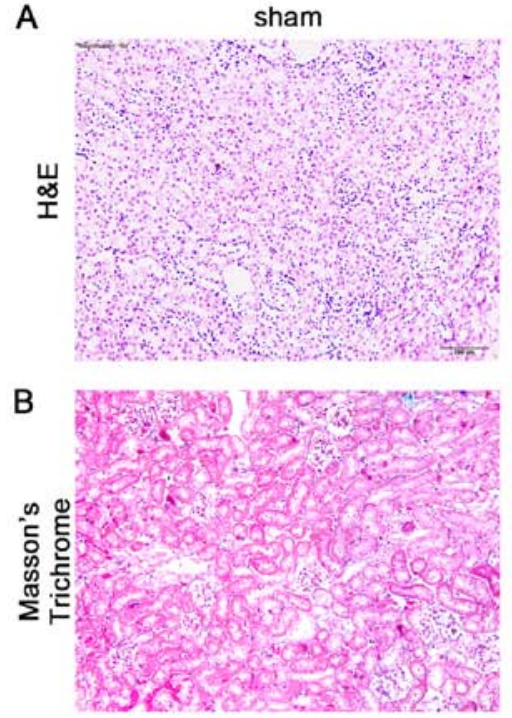

C

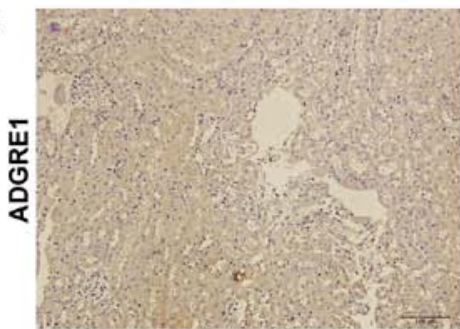

UUO day 7
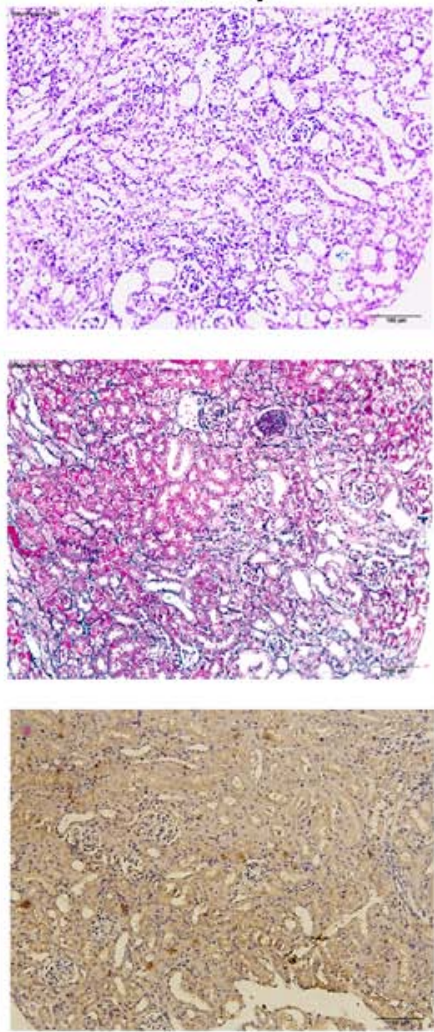
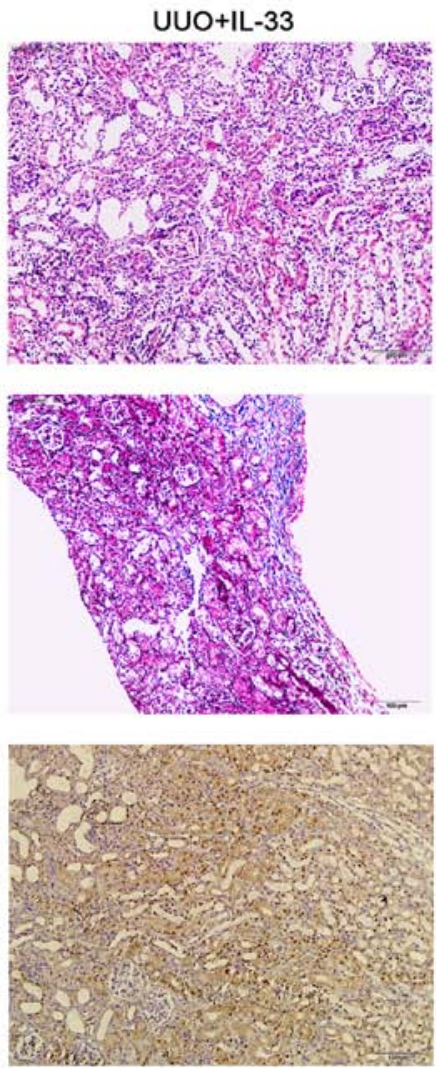

D
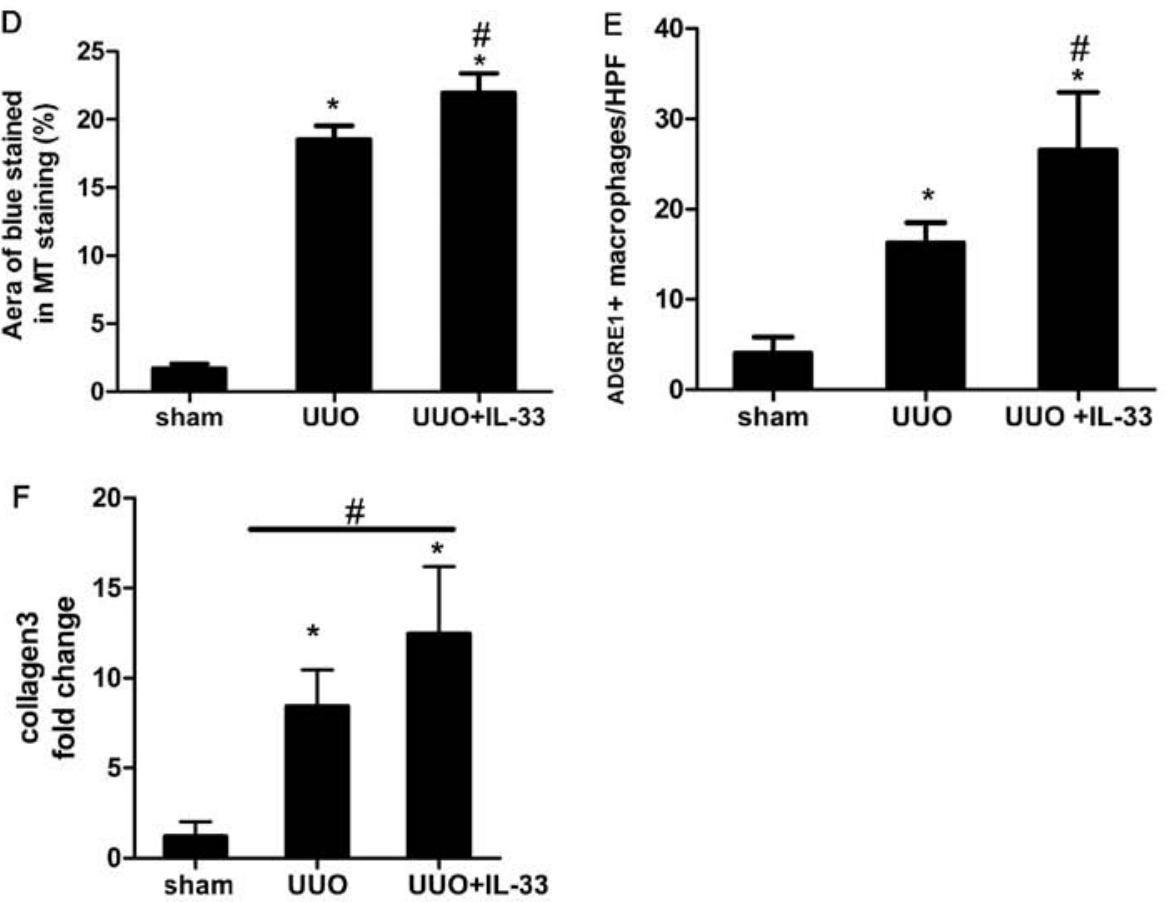

Figure 2. Exogenous mIL-33 increases UUO-induced renal tissue inflammation, fibrosis and macrophage infiltration. (A) H\&E staining suggested that mIL-33 increased UUO-induced inflammatory cell infiltration. (B) MT staining indicated that mIL-33 increased UUO-induced renal fibrosis. (C) Immunohistochemical staining of ADGRE1 suggested that mIL-33 increased UUO-induced renal tissue macrophage infiltration. (D) Quantification of MT staining results. (E) Quantification of ADGRE1 positive cells. (F) Collagen 3 relative mRNA expression levels. Mouse kidneys were harvested 7 days after surgery. Magnification, x200. Data are expressed as the mean \pm SD from three independent experiments. ${ }^{*} \mathrm{P}<0.05$ vs. sham; ${ }^{*} \mathrm{P}<0.05$ vs. UUO. H\&E, hematoxylin and eosin; mIL, mature interleukin; ADGRE1, adhesion G protein-coupled receptor E1; UUO, unilateral ureteral obstruction; MT, Masson's trichrome; HPF, high-power field.

inflammation and interstitial fibrosis (Fig. 3B, C, E and F), and abrogated the expression levels of IL- 13 and TGF- $\beta 1$ (Fig. 3I-L). Additionally, macrophage ablation decreased the expression levels of IL-33 and IL1RL1 (Fig. 3G and H). The present findings suggested that macrophages served an important role in the mechanism underlying IL-33/IL1RL1 role in promoting renal fibrosis through the production of fibrogenic factors, including TGF- $\beta 1$ and IL-13. 
A (ADGRE1) sham
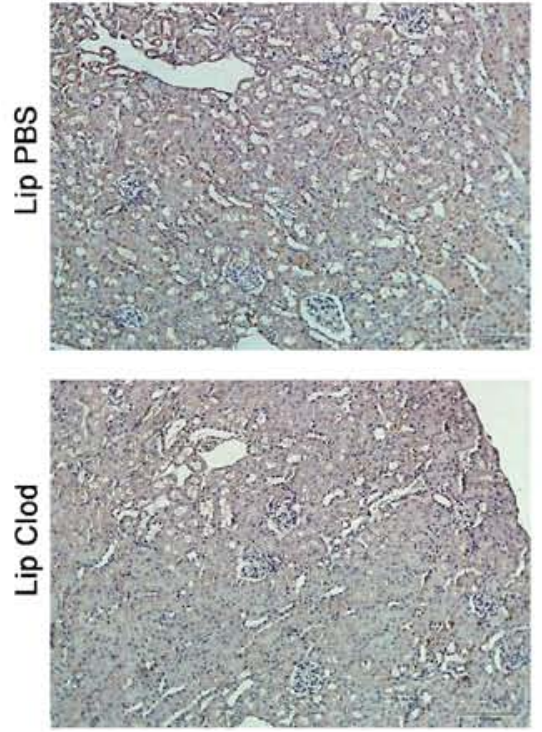

B (H\&E)
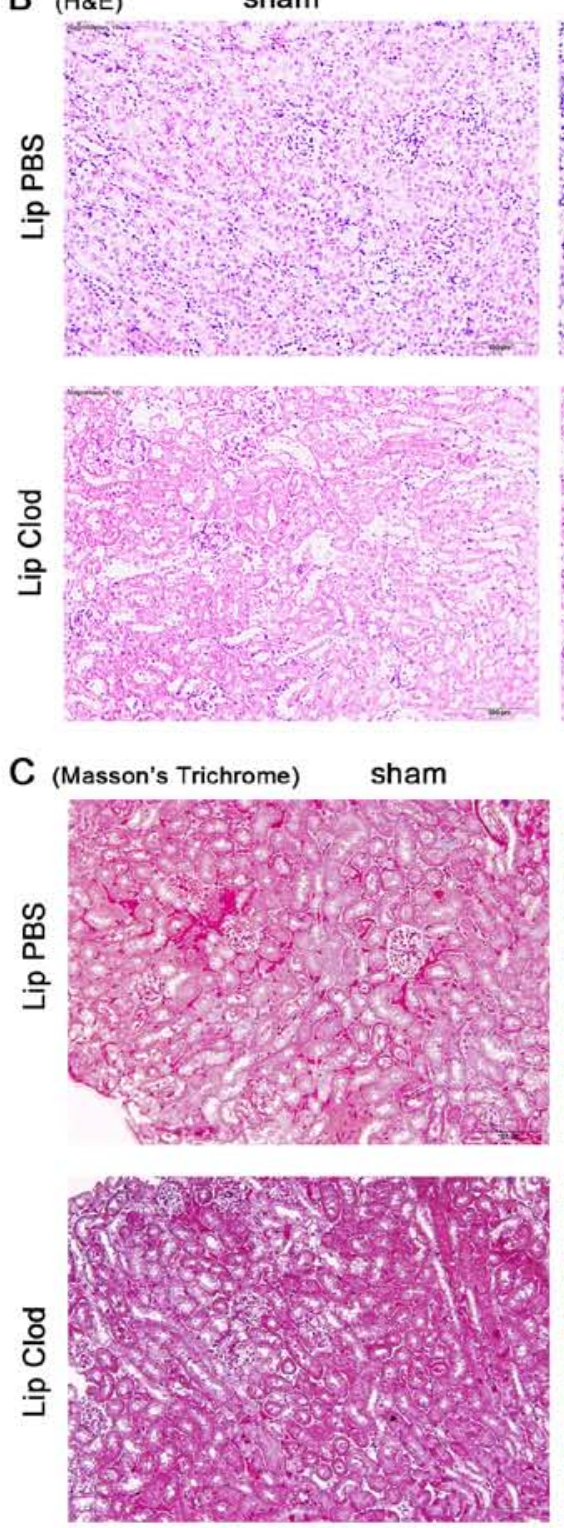

UUo
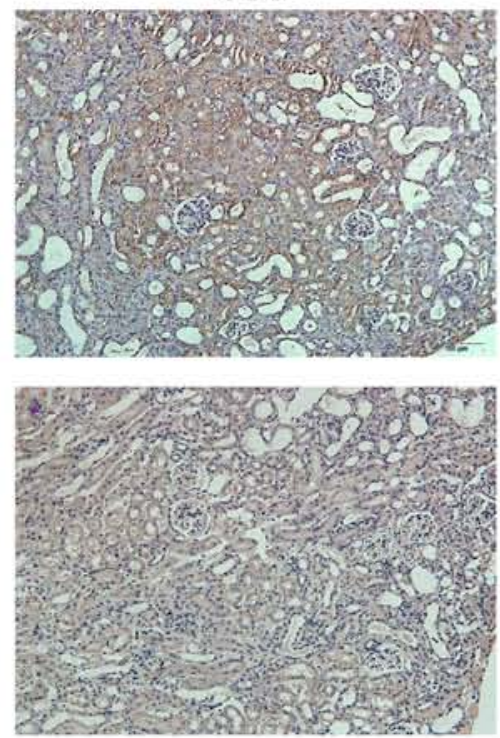

UUO
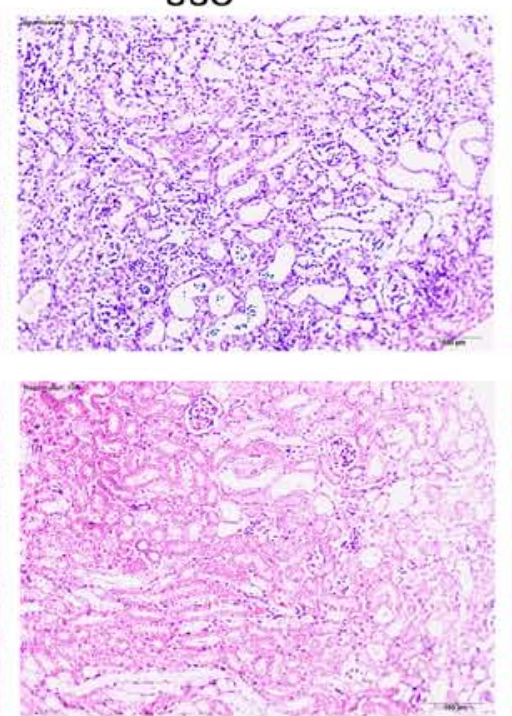

UUO
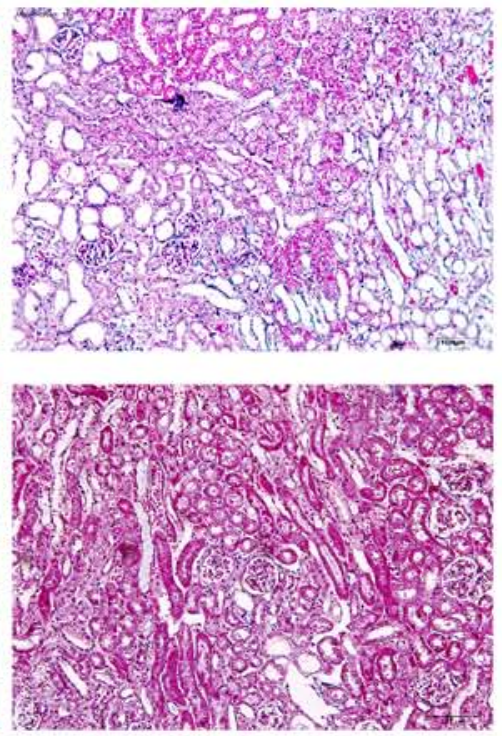

UUO+IL-33
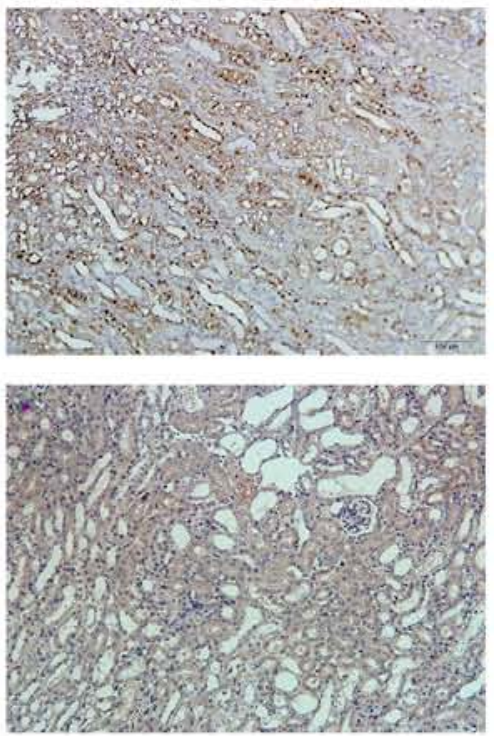

UUO+IL-33
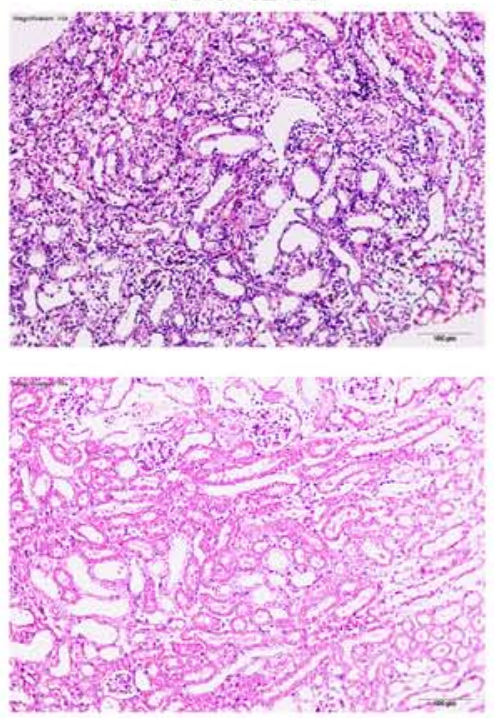

UUO+IL-33
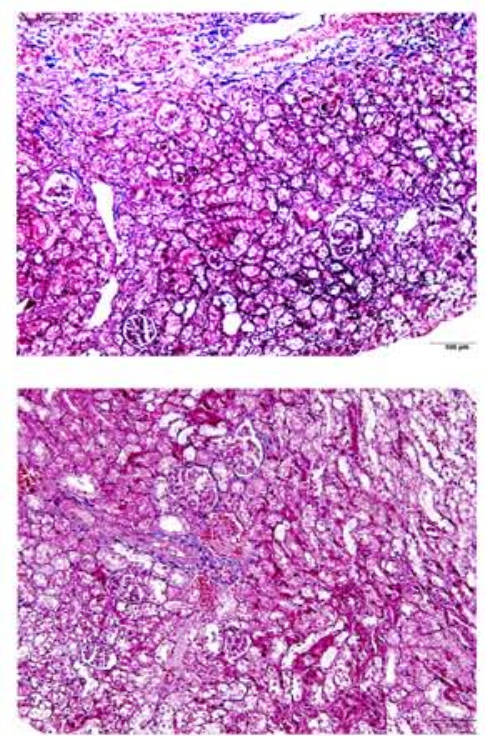

Figure 3. UUO induces interleukin IL-33, which promotes renal fibrosis via macrophages. Mice were subjected to sham surgery, UUO or UUO + IL-33, and treated with clodronate or control liposomes. Mouse kidneys were harvested 7 days after surgery. (A) Immunohistochemical staining of ADGRE1 suggested that macrophages were ablated by clodronate treatment. (B) H\&E staining suggested that macrophage depletion reduced UUO-induced inflammatory cell infiltration. (C) MT staining suggested that macrophage depletion reduced UUO-induced renal fibrosis. 

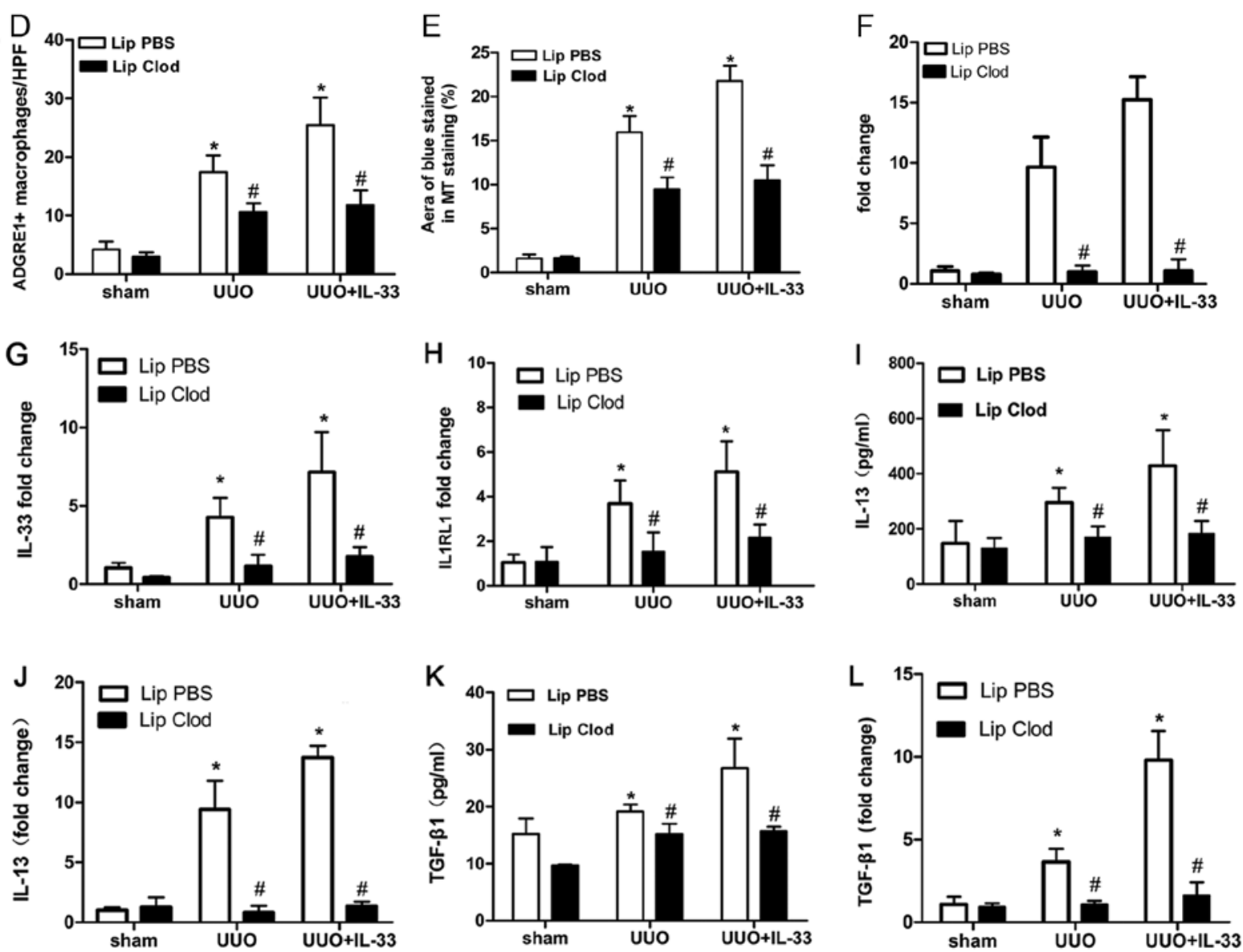

Figure 3. Continued. (D) Quantification of ADGRE1 positive cells. (E) Quantification of MT staining results. (F) Collagen 3 relative mRNA expression levels. (G) IL-33 relative mRNA expression levels. (H) IL1RL1 relative mRNA expression levels. (I) IL-13 serum levels. (J) IL-13 relative mRNA expression levels. (K) TGF- $\beta 1$ serum levels. (L) TGF- $\beta 1$ relative mRNA expression levels. Magnification, $x 200$. Data are expressed as the mean \pm SD from three independent experiments. ${ }^{*} \mathrm{P}<0.05$ vs. sham; ${ }^{\#} \mathrm{P}<0.05$ vs. respective Lip PBS group. H\&E, hematoxylin and eosin; ADGRE1, adhesion G protein-coupled receptor E1; UUO, unilateral ureteral obstruction; MT, Masson's trichrome; Lip, liposomes; Clod, clodronate; IL, interleukin; IL1RL1, interleukin 1 receptor like 1; TGF, transforming growth factor.

IL-33 increases UUO-induced renal fibrosis by promoting the polarization of macrophages to M2 phenotype. Previous in vitro studies demonstrated that IL-33 increased the IL-13-mediated macrophage polarization to M2 phenotype (25). In the present study, the mRNA expression level of ARG1, an M2 marker (32), was investigated. Treatment with IL-33 increased the mRNA expression of ARG1 in UUO mice (Fig. 4A). During UUO-induced renal fibrosis, the protein expression level of ARG1 increased compared with the sham-operated group, and exogenous IL-33 increased this effect (Fig. 4B).

\section{Discussion}

Although the IL-33/IL1RL1 pathway has been studied in skin, heart, liver and lung fibrosis (33), only a limited number of studies have investigated this pathway in kidney fibrosis. Upregulation of the IL-33/IL1RL1 signaling pathway in human serum may promote renal interstitial fibrosis; however, whether this process is related to macrophages remains unclear (23). Previous studies investigated the polarization of macrophages to M2 in the process of renal fibrosis $(24,34)$, but the mechanisms underlying macrophage polarization have yet to be fully elucidated. In the present study, IL-33 was identified to increase UUO-induced renal fibrosis in mice. The present results suggested that this effect was mediated by the production of IL-13 and TGF- $\beta 1$ by M 2 macrophages. These cytokines are powerful activators of fibroblasts, and are able to stimulate fibroblast proliferation and function, increasing collagen synthesis and inducing renal fibrosis $(35,36)$.

The present data suggested that the IL-33/IL1RL1/macrophage pathway is involved in the UUO-mediated renal fibrotic process. Compared with the control group, the expression levels of IL-33 and IL1RL1 in UUO-induced renal fibrotic tissues were significantly increased between days 3 and 14 following surgery. Moreover, the immunohistochemical and histopathological results of the kidney tissue analyses suggested that IL-33 was primarily synthesized in the renal tubule area. Treatment with mIL-33 increased renal fibrosis and macrophage infiltration induced by UUO. Additionally, mIL-33 increased the expression levels of IL-13 and TGF- $\beta 1$, suggesting that IL-33 may be a fibrogenic factor. Following macrophage ablation in the UOO group, the expression levels of IL-33 and its receptor, IL1RL1, were decreased to similar levels as the sham group. Additionally, renal fibrosis was decreased, and the serum levels of IL-13 and TGF- $\beta 1$ were 

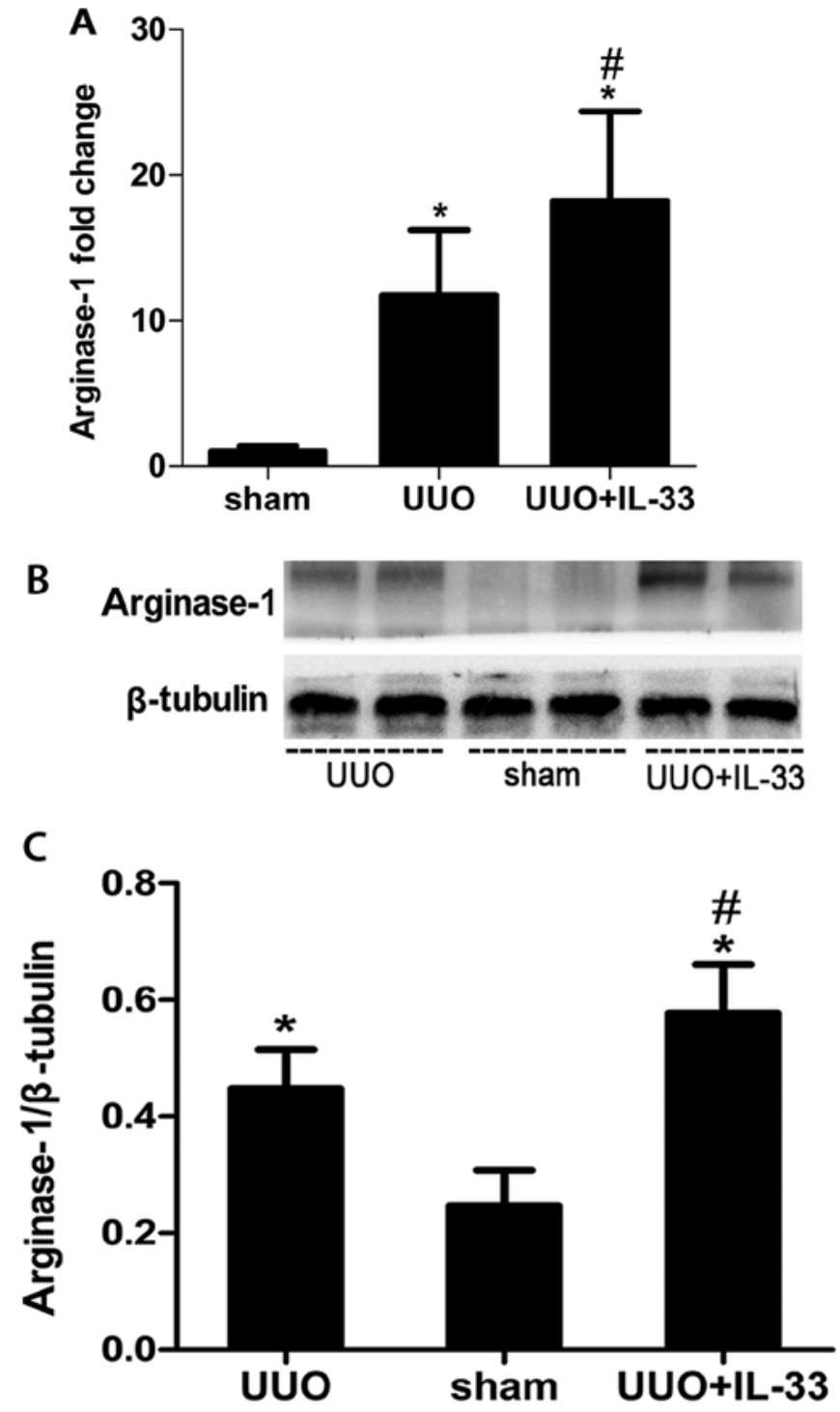

Figure 4. IL-33 polarizes M2 macrophages in renal fibrosis. Mice were subjected to sham surgery, UUO or UUO + IL-33. ARG1 is an M2 marker. (A) mRNA expression levels of ARG1 increased in UOO mice following IL-33 treatment. (B) Western blotting of ARG1 in UUO tissues. (C) Densitometric quantification suggested that the protein expression level of ARG1 was higher following UUO, and this effect was further increased by treatment with IL-33, suggesting that IL-33 polarized M2 macrophages during UUO. Data are expressed as the mean \pm SD from three independent experiments. ${ }^{~} \mathrm{P}<0.05$ vs. sham; ${ }^{\#} \mathrm{P}<0.05$ vs. UOO. UUO, unilateral ureteral obstruction; IL, interleukin; ARG1, arginase-1.

reduced. Furthermore, following macrophage ablation, treatment with mIL-33 was not sufficient to reverse these effects in UUO mice. Collectively, the present results suggested that the role of mIL-33/IL1RL1 in promoting fibrosis in UUO mice was primarily due to the action of macrophages and the resulting increase in fibrogenic cytokines.

Wang et al (37) found that the majority of macrophages expressed M2 markers, and only a small number expressed M1 markers during renal fibrosis. However, this previous study did not investigate the relationship between macrophages and IL-33 during renal fibrosis. Pan et al (24) demonstrated that macrophages recruited to the fibrotic region were polarized to the M2 subtype after renal injury in UUO mice. In acute fibrotic lesions, M1 inflammatory macrophages can transdifferentiate to M2 macrophages $(5,38)$. However, the mechanism of macrophage polarization into the M2 subtype during renal fibrosis is not clear. In the present study, IL-33 was identified to serve an important role in this process. During UUO-induced renal fibrosis, the expression level of ARG1, a surface marker of M2 macrophages, was significantly higher compared with the sham group, and this effect was further increased by treatment with IL-33. A previous in vitro study found that IL-33 increased the IL-13-mediated polarization of nonpolarized M0 macrophages into M2 macrophages (25). In addition, it was previously identified that in vitro-differentiated M2 macrophages express TGF- $\beta 1$, an important fibrogenic factor (39). The present study identified that the expression levels of IL-13, IL-33, ARG1 and TGF- $\beta 1$ increased during UUO-induced renal fibrosis, and that, in obstructive renal injury, IL-13 and IL-33 acted on recruited macrophages, polarizing them to the M2 phenotype. Additionally, M2 macrophages were previously found to synthesize TGF- $\beta 1$, which, together with IL-13, may promote the development of renal fibrosis (31).

The immune system serves an important role in the progression of CKD. Specifically, the stimulation of the immune system in the kidney can result in the recruitment and in the activation of immune cells. Dysregulated tissue repair processes are an important cause of fibrosis (40). During acute kidney injury (AKI) caused by sterile tissue damage, resident macrophages and dendritic cells are activated. Resident macrophages recruit more white blood cells and initiate the immune response to clear cell debris and dead tissue $(10,41)$. These repair mechanisms are important, but immune cells may prolong the inflammatory process in response to certain chemokines, exacerbating kidney damage (42). The present results suggested that, during UUO-induced renal fibrosis, the inflammatory cytokine IL-33 activated and polarized recruited macrophages, leading to the secretion of IL-13 and TGF- $\beta 1$, which were able to increase renal injury and fibrosis.

Current treatment methods for AKI and CKD involve supportive and alternative therapies. To the best of the authors' knowledge, targeted therapy to treat AKI and CKD has not yet been used in a clinical setting. A number of previous studies on experimental ischemic AKI demonstrated that cell therapy with dendritic and regulatory $\mathrm{T}$ cells was able to promote tissue repair $(43,44)$. Additionally, a previous study demonstrated the efficacy of treatments aimed to targets factors that are involved in the recruitment, activation, or are produced by macrophages (45). The present study investigating the mechanism underlying IL-33-mediated macrophages regulation may provide new insights into the development of novel therapeutic strategies to treat renal fibrosis.

\section{Acknowledgements}

The authors would like to thank Dr Dong Li (Department of Immunology, College of Basic Medicine, Jilin University) for assistance in animal breeding, drug treatment and design of the primers used in the qPCR experiments, and Dr Jinyan $\mathrm{Yu}$ (Department of Respiratory and Critical Care Medicine, The Second Hospital of Jilin University) for assistance in the immunohistochemical experiments. 


\section{Funding}

The present study was supported by The Natural Science Foundation from the Department of Science and Technology of Jilin Province (grant no. 20160101129JC), the Special Medical Foundation from The Department of Finance of Jilin Province (grant no. 2017 0125), and Jilin Province Science and Technology Plan Outstanding Youth Talent Fund Project (grant no. 20180520137JH).

\section{Availability of data and materials}

All data generated or analyzed for the present study are included in this published paper.

\section{Authors' contributions}

HL conceived and designed the experiments. YL performed the experiments and wrote the manuscript. JL provided reagents and materials, and analyzed the data. TY prepared the figures and performed the western blot analysis. BY interpreted the data. All authors have read and approved the final manuscript.

\section{Ethics approval and consent to participate}

All procedures were approved by The Animal Experimental Ethics Committee of Jilin University.

\section{Patient consent for publication}

Not applicable.

\section{Competing interests}

The authors declare that they have no competing interests.

\section{References}

1. Barnes JL and Glass WFII: Renal interstitial fibrosis: A critical evaluation of the origin of myofibroblasts. Contrib Nephrol 169: 73-93, 2011.

2. Hill NR, Fatoba ST, Oke JL, Hirst JA, O'Callaghan CA, Lasserson DS and Hobbs FD: Global prevalence of chronic kidney disease-A systematic review and meta-analysis. PLoS One 11: e0158765, 2016.

3. Zhao J, Wang L, Cao A, Jiang M, Chen X and Peng W: Renal tubulointerstitial fibrosis: A review in animal models. J Integr Nephrol Androl 2: 75-80, 2015.

4. Imig JD and Ryan MJ: Immune and inflammatory role in renal disease. Compr Physiol 3: 957-976, 2013.

5. Xiao X, Gaffar I, Guo P, Wiersch J, Fischbach S, Peirish L, Song Z, El-Gohary Y, Prasadan K, Shiota C and Gittes GK: M2 macrophages promote beta-cell proliferation by up-regulation of SMAD7. Proc Natl Acad Sci USA 111: E1211-E1220, 2014.

6. Chevalier RL, Forbes MS and Thornhill BA: Ureteral obstruction as a model of renal interstitial fibrosis and obstructive nephropathy. Kidney Int 75: 1145-1152, 2009.

7. Forbes MS, Thornhill BA and Chevalier RL: Proximal tubular injury and rapid formation of atubular glomeruli in mice with unilateral ureteral obstruction: A new look at an old model. Am J Physiol Renal Physiol 301: F110-F117, 2011.

8. Forbes MS, Thornhill BA, Minor JJ, Gordon KA, Galarreta Cl and Chevalier RL: Fight-or-flight: Murine unilateral ureteral obstruction causes extensive proximal tubular degeneration, collecting duct dilatation, and minimal fibrosis. Am J Physiol Renal Physiol 303: F120-F129, 2012.
9. Ferrario F, Castiglione A, Colasanti G, Barbiano di Belgioioso G, Bertoli S and D'Amico G: The detection of monocytes in human glomerulonephritis. Kidney Int 28: 513-519, 1985.

10. Meng XM, Nikolic-Paterson DJ and Lan HY: Inflammatory processes in renal fibrosis. Nat Rev Nephrol 10: 493-503, 2014.

11. Kim MG, Kim SC, Ko YS, Lee HY, Jo SK and Cho W: The role of M2 macrophages in the progression of chronic kidney disease following acute kidney injury. PLoS One 10: e0143961, 2015.

12. Mosser DM and Edwards JP: Exploring the full spectrum of macrophage activation. Nat Rev Immunol 8: 958-969, 2008.

13. Guiteras R, Flaquer M and Cruzado JM: Macrophage in chronic kidney disease. Clin Kidney J 9: 765-771, 2016.

14. Guiteras R, Sola A, Flaquer M, Hotter G, Torras J, Grinyó JM and Cruzado JM: Macrophage overexpressing NGAL ameliorated kidney fibrosis in the UUO mice model. Cell Physiol Biochem 42: 1945-1960, 2017.

15. Colin S, Chinetti-Gbaguidi G and Staels B: Macrophage phenotypes in atherosclerosis. Immunol Rev 262: 153-166, 2014.

16. Flavell RA, Sanjabi S, Wrzesinski SH and Licona-Limón P: The polarization of immune cells in the tumour environment by TGFbeta. Nat Rev Immunol 10: 554-567, 2010.

17. Liew FY, Girard JP and Turnquist HR: Interleukin-33 in health and disease. Nat Rev Immunol 16: 676-689, 2016.

18. Ricardo SD, van Goor H and Eddy AA: Macrophage diversity in renal injury and repair. J Clin Invest 118: 3522-3530, 2008.

19. Wynn TA and Ramalingam TR: Mechanisms of fibrosis: Therapeutic translation for fibrotic disease. Nat Med 18: 1028-1040, 2012.

20. Lott JM, Sumpter TL and Turnquist HR: New dog and new tricks: Evolving roles for IL-33 in type 2 immunity. J Leukoc Biol 97: 1037-1048, 2015.

21. Kurowska-Stolarska M, Hueber A, Stolarski B and McInnes IB: Interleukin-33: A novel mediator with a role in distinct disease pathologies. J Intern Med 269: 29-35, 2011.

22. Staurengo-Ferrari L, Trevelin SC, Fattori V, Nascimento DC, de Lima KA, Pelayo JS, Figueiredo F, Casagrande R, Fukada SY, Teixeira MM, et al: Interleukin-33 receptor (ST2) deficiency improves the outcome of staphylococcus aureus-induced septic arthritis. Front Immunol 9: 962, 2018.

23. Chen WY, Chang YJ, Su CH, Tsai TH, Chen SD, Hsing CH and Yang JL: Upregulation of Interleukin-33 in obstructive renal injury. Biochem Biophys Res Commun 473: 1026-1032, 2016.

24. Pan B, Liu G, Jiang Z and Zheng D: Regulation of renal fibrosis by macrophage polarization. Cell Physiol Biochem 35: 1062-1069, 2015.

25. Li D, Guabiraba R, Besnard AG, Komai-Koma M, Jabir MS, Zhang L, Graham GJ, Kurowska-Stolarska M, Liew FY, McSharry C and Xu D: IL-33 promotes ST2-dependent lung fibrosis by the induction of alternatively activated macrophages and innate lymphoid cells in mice. J Allergy Clin Immunol 134: 1422-1432.e11, 2014.

26. Inada T, Yamanouchi Y, Jomura S, Sakamoto S, Takahashi M, Kambara T and Shingu K: Effect of propofol and isoflurane anaesthesia on the immune response to surgery. Anaesthesia 59: 954-959, 2004

27. Zhang WL, Liu MY, Zhang ZC and Duan CY: Effect of different anesthesia methods on erythrocyte immune function in mice. Asian Pac J Trop Med 6: 995-998, 2013.

28. Hohlbaum K, Bert B, Dietze S, Palme R, Fink H and Thöne-Reineke C: Severity classification of repeated isoflurane anesthesia in C57BL/6JRj mice-Assessing the degree of distress. PLoS One 12: e0179588, 2017.

29. van Rooijen $\mathrm{N}$ and Hendrikx E: Liposomes for specific depletion of macrophages from organs and tissues. Methods Mol Biol 605: $189-203,2010$

30. Ferenbach DA, Sheldrake TA, Dhaliwal K, Kipari TM, Marson LP, Kluth DC and Hughes J: Macrophage/monocyte depletion by clodronate, but not diphtheria toxin, improves renal ischemia/reperfusion injury in mice. Kidney Int 82: 928-933, 2012.

31. Aimo A, Migliorini P, Vergaro G, Franzini M, Passino C, Maisel A and Emdin M: The IL-33/ST2 pathway, inflammation and atherosclerosis: Trigger and target? Int J Cardiol 267: 188-192, 2018.

32. Sanson M, Distel E and Fisher EA: HDL induces the expression of the M2 macrophage markers arginase 1 and Fizz-1 in a STAT6-dependent process. PLoS One 8: e74676, 2013.

33. Molofsky AB, Savage AK and Locksley RM: Interleukin-33 in tissue homeostasis, injury, and inflammation. Immunity 42 : 1005-1019, 2015. 
34. Yu CC, Chien CT and Chang TC: M2 macrophage polarization modulates epithelial-mesenchymal transition in cisplatin-induced tubulointerstitial fibrosis. Biomedicine (Taipei) 6: 5, 2016.

35. Biernacka A, Dobaczewski $M$ and Frangogiannis NG: TGF- $\beta$ signaling in fibrosis. Growth Factors 29: 196-202, 2011.

36. Ramalingam TR, Gieseck RL, Acciani TH, M HartK, Cheever AW Mentink-Kane MM, Vannella KM and Wynn TA: Enhanced protection from fibrosis and inflammation in the combined absence of IL-13 and IFN- $\gamma$. J Pathol 239: 344-354, 2016.

37. Wang S, Meng XM, Ng YY, Ma FY, Zhou S, Zhang Y, Yang C, Huang XR, Xiao J, Wang YY, et al: TGF- $\beta /$ Smad3 signalling regulates the transition of bone marrow-derived macrophages into myofibroblasts during tissue fibrosis. Oncotarget 7: 8809-8822, 2016.

38. Ikezumi Y, Suzuki T, Yamada T, Hasegawa H, Kaneko U, Hara M, Yanagihara T, Nikolic-Paterson DJ and Saitoh A: Alternatively activated macrophages in the pathogenesis of chronic kidney allograft injury. Pediatr Nephrol 30: 1007-1017, 2015.

39. Lu J, Cao Q, Zheng D, Sun Y, Wang C, Yu X, Wang Y, Lee VW, Zheng G, Tan TK, et al: Discrete functions of M2a and M2c macrophage subsets determine their relative efficacy in treating chronic kidney disease. Kidney Int 84: 745-755, 2013.
40. Tecklenborg J, Clayton D, Siebert S and Coley SM: The role of the immune system in kidney disease. Clin Exp Immunol 192: 142-150, 2018.

41. Yatim KM and Lakkis FG: A brief journey through the immune system. Clin J Am Soc Nephrol 10: 1274-1281, 2015.

42. Kurts C, Panzer U, Anders HJ and Rees AJ: The immune system and kidney disease: Basic concepts and clinical implications. Nat Rev Immunol 13: 738-753, 2013.

43. Li L, Huang L, Ye H, Song SP, Bajwa A, Lee SJ, Moser EK, Jaworska K, Kinsey GR, Day YJ, et al: Dendritic cells tolerized with adenosine A2AR agonist attenuate acute kidney injury. J Clin Invest 122: 3931-3942, 2012.

44. Lai LW, Yong KC and Lien YH: Pharmacologic recruitment of regulatory $\mathrm{T}$ cells as a therapy for ischemic acute kidney injury. Kidney Int 81: 983-992, 2012.

45. Sean Eardley K and Cockwell P: Macrophages and progressive tubulointerstitial disease. Kidney Int 68: 437-455, 2005. 